DDS - Die Deutsche Schule

113. Jahrgang 2021, Heft 4, S. 396-408

https://doi.org/10.31244/dds.2021.04.03

CC BY-NC-ND 4.0

Waxmann 2021

Wolfram Cremer \& Antonia Tobisch

\title{
Übergänge in weiterführende Schulformen aus (verfassungs)rechtlicher Perspektive ${ }^{1}$
}

\section{Zusammenfassung $^{1}$}

Wie viel Freiheit Eltern sowie Schüler*innen im Hinblick auf die weiterführende Schulform nach der Grundschule gelassen werden sollte, ist schulpolitisch umstritten. Das schlägt sich in einer großen Vielfalt von Übergangsmodellen in den einzelnen deutschen Bundesländern nieder. Der folgende Beitrag bietet zunächst einen Überblick über die Rechtssituation in den verschiedenen Bundesländern. Im Anschluss werden die unterschiedlichen Regelungsmodelle unter Einbeziehung von Erkenntnissen der Bildungsforschung einer Bewertung am Maßstab des Grundgesetzes unterzogen.

Schlüsselwörter: Bildungsrecht, Bildungsmonitoring, Übergänge

\section{Transitions from Primary to Secondary School from a Constitutional Perspective}

\begin{abstract}
How much freedom parents and pupils should have with regard to the secondary school form after primary school is strongly controversial in terms of school politics. This is reflected in a wide variety of regulatory models in the individual federal states. The following article first provides an overview of the legal situation in the various federal states of Germany. Subsequently, the different regulatory models, taking into account the findings of educational research, will be subjected to an assessment on the scale of the German constitution.
\end{abstract}

Keywords: educational law, educational monitoring, transitions

1 Der Artikel schließt an den von Wolfram Cremer in dem Sammelwerk "Selektion und Gerechtigkeit in der Schule" (Institut für Bildungsforschung und Bildungsrecht e. V., 2012) veröffentlichten Beitrag „Die verbindliche Übergangsempfehlung zur Sekundarstufe zwischen Verfassungsauftrag und Verfassungswidrigkeit“ an (ebd., S. 79-108). 


\section{A Die Regelung des Übergangsregimes in den 16 Bundesländern}

Die in den 16 Bundesländern vorhandenen Regelungen zum Übergang in die Sekundarstufe werden in der Bildungsforschung regelmäßig zwei Grundtypen zugeordnet, der verbindlichen oder der unverbindlichen Übergangsempfehlung (vgl. nur Bellenberg \& Tillmann, 2011, S. 62 f.). Diese Unterscheidung wird auch hier zugrunde gelegt; indes wird hier ein dritter Grundtyp eingeführt, nämlich das sog. Beratungsmodell mit Präklusionswirkung. Dabei stellt die Zuordnung einer landesrechtlichen Regelung als verbindlich oder unverbindlich (resp. als Beratungsmodell mit Präklusionswirkung) hier prinzipiell auf den Zeitpunkt des erstmaligen Wechsels von der Primar- in die Sekundarstufe ab. Damit schließt die Einstufung als „unverbindlich“ nach der hier vorgenommenen Kategorisierung nicht aus, dass im Anschluss an eine allein den Eltern zugebilligte Schulformwahl in einem ersten Schritt (i.d.R. nach Klasse 4), verbindliche Versetzungen an eine andere (niedrigere) Schulform in einem zweiten Schritt (insbesondere nach Klasse 6) erfolgen können.

\section{Verbindliche Grundschulempfehlungen}

Verbindliche Grundschulempfehlungen beim Übergang in die Sekundarstufe sehen die Bundesländer Bayern, Brandenburg und Thüringen vor. Das gilt freilich nur insoweit, als die Eltern abweichend von der Empfehlung für den Besuch einer höheren Schulform votieren. Die grundsätzliche Verbindlichkeit der Grundschulempfehlung kann jeweils (mit leichten Abweichungen in der jeweiligen Ausgestaltung) nur durch das erfolgreiche Bestehen eines Probeunterrichts oder, im Fall von Bayern und Thüringen, durch das Erreichen gewisser Notenstufen überwunden werden. Details hier$\mathrm{zu}$ sind den $\$ \$ 2$ f. BayRSO, $\$ \$ 2$ f. BayGSO, $\$ 53$ BbgSchulG, $\$ \$ 40 \mathrm{ff}$. Bbg. Sek I-VO, $\$ 7$ Abs. 2 ThürSchulG sowie $\$ \$ 125,131$ ThürSchulO zu entnehmen.

\section{Unverbindliche Grundschulempfehlungen}

In Baden-Württemberg, Niedersachsen, dem Saarland und in Sachsen-Anhalt und obliegt die Schulformwahl allein den Eltern. Es finden zwar (teils obligatorische) Beratungsgespräche statt. Die Erziehungsberechtigten sind jedoch bei der Anmeldung ihres Kindes an die Empfehlungen in den Beratungsgesprächen nicht gebunden. Dies ergibt sich aus den $₫ \$ 6$ Abs. 5 und 59 NdsSchulG, aus $₫ 5$ Abs. 2 SchulG BW, $₫ 3$ AufnVO BW, aus $₫ 34$ SchulG LSA, $₫ 1$ Sek-I-Üg-VO LSA und aus $₫ 16$ SaarlZVOGS.

In Berlin, Hamburg, Hessen, Mecklenburg-Vorpommern, Nordrhein-Westfalen, Rheinland-Pfalz und Schleswig-Holstein dürfen zwar grundsätzlich die Eltern die weiter- 
führende Schulform frei wählen. Es gibt allerdings hier die Einschränkung, dass bei Besuch einer höheren Schule entgegen der Empfehlung eine gewisse Probezeit gilt (in der Regel die 5. und 6. Jahrgangsstufe), in der die Schüler*innen ihre tatsächliche Eignung für diese Schulform zeigen und sich bewähren müssen. Wird in der Probezeit eine entsprechende Eignung nicht festgestellt, so kann eine Querversetzung in eine niedrigere Schulform erfolgen. Einzelheiten sind $₫ 56$ BerlSchulG, $₫ 42 \mathrm{Hamb}$ SchulG, $\$ \$ 75$ Abs. 3, 77 HessSchulG, $\$ 66$ SchulG MV, $\$ \$ 9$ Abs. 3, 24 SchulG SH, $\S \$ 12$ Abs. 2, 20 f., 30 ÜSchulO Rh.-Pf. und $\$ \$ 11$ Abs. 5, 13 SchulG NRW zu entnehmen.

\section{Beratungsmodelle mit Präklusionswirkung}

In Bremen und Sachsen finden sich schließlich Regelungen zum Übergang von der Primar- in die Sekundarstufe, die sich einer „schlichten“ Zuordnung als verbindlich/ unverbindlich entziehen. Vielmehr enthalten die Schulgesetze einen hier als Beratungsmodell mit Präklusionswirkung firmierenden Regelungsmodus.

In Bremen findet nach dem 1. Halbjahr der 4. Jahrgangsstufe ein verbindliches Beratungsgespräch mit den Erziehungsberechtigten über den weiteren Bildungsgang statt ( $\$ 37$ a BremSchulG, $₫ 13$ GrSchulOrgV BR). Grundsätzlich wählen die Erziehungsberechtigten den weiteren Bildungsgang für ihr Kind. Nehmen die Erziehungsberechtigten allerdings nicht an der verbindlichen Beratung teil, weist die Grundschule die Schüler ${ }^{\star}$ innen einer Schulart zu ( $\$ 37$ a BremSchulG).

In Sachsen dürfen die Eltern gem. $\$ 34$ Abs. 1 Satz 1 SächsSchulG zwar grundsätzlich die weiterführende Schule wählen. Melden Eltern ihr Kind jedoch entgegen der Bildungsempfehlung an einem Gymnasium an, wird durch das Gymnasium ein Beratungsgespräch vereinbart und bei der Einladung auf die Folgen des Nichterscheinens hingewiesen ( $\$ 34$ Abs. 2 Satz 2 SächsSchulG). Erscheint ohne wichtigen Grund kein Elternteil zum vereinbarten Beratungsgespräch, gilt die Anmeldung als zurückgenommen ( $\$ 34$ Abs. 2 Satz 4 SächsSchulG). Aber auch, wenn das Beratungsgespräch stattfindet, erfolgt eine Aufnahme am Gymnasium nur, wenn die Eltern innerhalb von drei Wochen schriftlich mitteilen, dass sie an der Anmeldung festhalten wollen, sofern nicht im Gespräch eine Empfehlung zum Besuch eines Gymnasiums erteilt wird (\$ 6 Abs. 1 Satz 2 Nr. 2 SächsSOGYA). 
Übergänge in weiterführende Schulformen aus (verfassungs)rechtlicher Perspektive |

\section{B Verfassungsmäßigkeit verbindlicher Übergangsempfehlungen}

Im Anschluss an diese Kategorisierung der verschiedenen Übergangsregimes wird nunmehr zunächst nach der Verfassungsmäßigkeit verbindlicher Übergangsentscheidungen gefragt. Dabei soll hier eine Verengung des Prüfungsmaßstabs dahingehend erfolgen, dass die neben dem Grundgesetz für die Rechtmäßigkeit der Schulgesetzgebung der Länder maßgeblichen Landesverfassungen außer Betracht bleiben. Dieses wird dadurch legitimiert, dass sich aus den Landesverfassungen - soweit ersichtlich - für diesen Zusammenhang keine über das Grundgesetz hinausgehenden Vorgaben für die Schulgesetzgebung ergeben bzw. im Übrigen eine grundgesetzkonforme Auslegung erfolgen müsste (vgl. dazu Barczak, 2011, S. 126 f.; Meinel, 2007, S. 67 f.).

\section{Zum einschlägigen Verfassungsrecht}

Zentral für einen etwaigen Verfassungsrechtsverstoß im Falle eines Nichtübereinstimmens von verbindlicher Übergangsentscheidung und Elternwunsch ist das Elternrecht aus Art. 6 Abs. 2 Satz 1 GG, wonach „Pflege und Erziehung der Kinder [...] das natürliche Recht der Eltern und die ihnen zuvörderst obliegende Pflicht“ sind. Verstärkt wird das Elternrecht durch verschiedene Grundrechtspositionen des betroffenen Kindes (vgl. dazu Tettinger, 2005, S. 333), jedenfalls soweit dessen Wunsch mit dem Elternwunsch übereinstimmt. $\mathrm{Zu}$ nennen ist vor allem die in Art. 12 Abs. 1 GG geschützte Berufsfreiheit (vgl. dazu Scholz, 2020, Rn. 188; a. A. Beaucamp, 2009, S. 281). Neben Art. 12 Abs. 1 GG wird das Allgemeine Persönlichkeitsrecht der Schülerinnen gem. Art. 2 Abs. 1 i.V.m. Art. 1 Abs. 1 GG durch hoheitliche Übergangsempfehlungen, ob verbindlich oder unverbindlich, berührt (a. A. wohl Meinel, 2007, S. 70). Letztlich kommt dem Schüler*innenwunsch und den korrespondierenden Grundrechtspositionen freilich neben dem Elternrecht regelmäßig keine entscheidende verfassungsrechtliche Bedeutung zu. Den Eltern kommt prinzipiell und nicht nur gegenüber dem Staat (so bereits BVerfGE 4, 52 (56)), sondern auch gegenüber ihren noch nicht grundrechtsmündigen ${ }^{2}$ Kindern ein Interpretationsprimat zur Bestimmung des Wohls eben dieser, ihrer Kinder, zu (Roth, 2003, S. 141). Dabei darf der Wille der Kinder von Verfassungs wegen in diesem Konflikt gleichwohl nicht gänzlich unberücksichtigt bleiben (Hölbling, 2010, S. 146 ff.).

Bleibt zu fragen, welche verfassungsrechtlich relevanten Positionen dem Elterngrundrecht und den dieses regelmäßig verstärkenden Schüler*innengrundrechten entgegenstehen. Generell findet das Elterngrundrecht aus Art. 6 Abs. 2 S. 1 GG erst in Art. 6 Abs. 2 S. 2 GG und dem dort statuierten staatlichen Wächteramt seine Grenze (Jeand'Heur, 1993). Für den Schulbereich gilt freilich zumindest partiell anderes,

2 Grundrechtsmündigkeit bezeichnet nach verbreitetem Verständnis die Fähigkeit, eigene Rechte selbst gerichtlich durchzusetzen. 
denn in Art. 7 Abs. 1 GG heißt es: „Das gesamte Schulwesen steht unter der Aufsicht des Staates“. Daraus leiten BVerfG und die ganz herrschende Meinung (h. M.) zunächst einmal ganz grundsätzlich eine Befugnis (und Pflicht) des Staates zur Organisation, Leitung und Planung des Schulwesens ab (vgl. nur BVerfGE 26, 228 (238)). Daraus folgt z. B., dass Eltern (und Kinder) das landesrechtliche Angebot an Schulformen - soweit es nicht grob interessenwidrig ausgestaltet ist, etwa jegliche Differenzierung bis zum Ende der Schullaufbahn vermissen lässt (so mindestens implizit BVerfGE 45, 400 (415 f.)) - akzeptieren müssen. Art. 6 Abs. 2 GG lässt sich der Gedanke entnehmen, dass der Staat dort, wo er die elterliche Entscheidung im Einzelfall korrigiert bzw. substituiert, einer besonderen Rechtfertigung bedarf. Es gilt angesichts Art. 7 Abs. 1 GG (staatliche Schulhoheit) zwar nicht die hohe Interventionsschwelle des Art. 6 Abs. 2 Satz 2 GG (staatliches Wächteramt, vgl. BVerfGE 34, 165 [183)), wohl aber vermag Art. 7 Abs. 1 GG allein den Grundrechtseingriff in Art. 6 Abs. 2 Satz 1 GG (Elternverantwortung) nicht zu legitimieren (vgl. auch Meinel, 2007, S. 68 ff.). In diesen Kontext gehört auch die Schulformwahl. Diesbezüglich gilt es zu beachten, dass der Staat mit der Statuierung verbindlicher Übergangsentscheidungen möglicherweise auch die Grundrechte (potenzieller) Mitschüler ${ }^{\star}$ innen, namentlich Art. 12 Abs. 1 GG (Berufsfreiheit), durch das Fernhalten ungeeigneter Schüler*innen schützt.

Bei einer verfassungsrechtlichen Würdigung ist neben den genannten Aspekten auch ein Verstoß gegen das Gleichheitsgebot, insbesondere eine Verletzung des Art. 3 Abs. 3 Satz 1 6. Alt. GG (Diskriminierungsverbot aufgrund der Herkunft), in Betracht zu ziehen. Die Prüfung einer Verletzung des Elterngrundrechts und eines Verstoßes gegen das Gleichheitsgebot stehen dabei nicht unverbunden nebeneinander, denn ein Verstoß gegen Art. 3 Abs. 3 Satz 1 6. Alt. GG begründete nach Maßgabe der ElfesDoktrin zugleich eine Verletzung von Art. 6 Abs. 2 Satz 1 GG (Elternverantwortung) und ggf. der korrespondierenden Grundrechte der Kinder. Aus der sog. Elfes-Doktrin (BVerfGE 6, 32 ff.) geht der umfassende Abwehrgehalt der Freiheitsgrundrechte und damit die Forderung hervor, dass ein grundrechtseinschränkendes Gesetz in jeder Hinsicht verfassungsmäßig sein muss (näher hierzu Frotz, 2006, S. 45 ff.). Ein wegen Verstoßes gegen Art. 3 GG verfassungswidriges Gesetz kann demnach nicht als rechtmäßige Schrankenbestimmung fungieren.

\section{Anwendung der verfassungsrechtlichen Maßstäbe}

Nachdem nun die relevanten Verfassungsgüter ausgemacht wurden, gilt es, diese auf die Übergangsmodelle mit verbindlicher Übergangsempfehlung anzuwenden. Insofern muss zwischen einer sog. Positivkorrektur der elterlichen Entscheidung, wenn das Kind entgegen dem Willen der Eltern eine höhere Schulform besuchen muss, und 
Übergänge in weiterführende Schulformen aus (verfassungs)rechtlicher Perspektive |

einer sog. Negativkorrektur der elterlichen Entscheidung unterschieden werden, wenn das Kind entgegen dem Willen der Eltern eine niedrigere Schulform besuchen muss.

\section{Positivkorrektur der elterlichen Entscheidung}

Eine Positivkorrektur der elterlichen Entscheidung beeinträchtigt ihr Elternrecht aus Art. 6 Abs. 2 Satz 1 GG. Es stellt sich die Frage nach einer verfassungsrechtlichen Rechtfertigung.

a) Begründungsansätze für die verfassungsrechtliche Zulässigkeit einer Positivkorrektur

aa) Unterforderung der Schülerin bzw. des Schülers

Für eine staatliche Positivkorrektur des Elternwillens lässt sich vor allem anführen, dass es nicht hinnehmbar ist, die Begabung eines Kindes durch Unterforderung verkümmern zu lassen resp. ihm angesichts der faktisch geringen Durchlässigkeit der Schulsysteme in Deutschland einen begabungsgerechten Bildungs- und Ausbildungsweg vorzuenthalten. Indes hat das BVerfG ausgeführt, dass der Staat zwar die Aufgabe hat,

„im Rahmen seiner finanziellen und organisatorischen Möglichkeiten ein Schulsystem bereitzustellen, das den verschiedenen Begabungsrichtungen Raum zur Entfaltung lässt, sich aber von jeder ,Bewirtschaftung des Begabungspotentials freihält“ (BVerfGE 34, 165 (184)).

Und weiter heißt es:

„Die Entscheidung über den weiteren Bildungsweg des Kindes hat das Grundgesetz zunächst den Eltern als den natürlichen Sachwaltern für die Erziehung des Kindes belassen. Damit wird jedenfalls dem Grundsatz nach berücksichtigt, daß sich das Leben des Kindes nicht nur nach seiner ohnehin von den Umweltfaktoren weitgehend geprägten Bildungsfähigkeit und seinen Leistungsmöglichkeiten gestaltet, sondern daß hierfür auch die Interessen und Sozialvorstellungen der Familie von großer Bedeutung sind. Diese primäre Entscheidungszuständigkeit der Eltern beruht auf der Erwägung, daß die Interessen des Kindes am besten von den Eltern wahrgenommen werden. Dabei wird sogar die Möglichkeit in Kauf genommen, daß das Kind durch einen Entschluß der Eltern Nachteile erleidet, die im Rahmen einer nach objektiven Maßstäben betriebenen Begabtenauslese vielleicht vermieden werden könnten. Dieses Bestimmungsrecht der Eltern umfaßt auch die Befugnis, den von ihrem Kind einzuschlagenden Bildungsweg in der Schule frei zu wählen" (ebd.). 
In der Tat lässt sich Bezug nehmend auf obige Überlegungen für einen weitgehenden Primat des Elternwillens anführen, dass es bei der Schulwahl - eben anders als bei der Organisation, Planung und Leitung im Schulwesen - um das Einzelschicksal ihres Kindes geht (vgl. Maaz et al., 2019, S. 17). Über dieses Schicksal zu bestimmen, ist grundsätzlich das Recht der Eltern, welches nicht schon allein durch den Hinweis auf die mangelnde Begabungsadäquanz der elterlichen Entscheidung staatlicherseits substituiert werden darf.

\section{bb) Hemmung der Mitschüler*innen}

Anknüpfungspunkt für die verfassungsrechtliche Rechtfertigung könnte ferner die These sein, dass das leistungsstärkere Kind etwa eine Gymnasialempfehlung erhalten hat, dessen Eltern aber für die Hauptschule optieren, seine leistungsschwächeren Mitschüler*innen überfordert, frustriert, demotiviert und hemmt, indem es ihnen stets deutlich voraus ist. Indes fehlt es - soweit ersichtlich - an Belegen für eine solche These aus der Bildungsforschung. Sie bleibt also in der Sache Spekulation und vermag demgemäß auch keinen Anknüpfungspunkt für eine verfassungsrechtliche Rechtfertigung zu bilden.

\section{b) Ergebnis}

Eine in das Elterngrundrecht eingreifende Positivkorrektur, welche freilich in ,Reinform' in keinem Bundesland vorgesehen ist, lässt sich verfassungsrechtlich nicht rechtfertigen. Angesichts des Befundes zur verfassungsrechtlichen Bewertung einer Positivkorrektur muss das oben geschilderte, in Bremen praktizierte Beratungsmodell mit Präklusionswirkung als verfassungswidrig gekennzeichnet werden, führt es doch letztendlich zu einer eben verfassungswidrigen Positivkorrektur - geschieht dies auch nur gegenüber nachhaltig unkooperativen oder desinteressierten Eltern.

\section{Negativkorrektur der elterlichen Entscheidung}

a) Verstoß gegen Art. 6 Abs. 2 Satz 1 GG

Auch hinsichtlich der Negativkorrektur einer elterlichen Entscheidung ist zunächst an eine Verletzung von Art. 6 Abs. 2 Satz 1 GG (Elternverantwortung) zu denken. 
Übergänge in weiterführende Schulformen aus (verfassungs)rechtlicher Perspektive | aa) Überforderung der Schülerin bzw. des Schülers

In dieser Konstellation geht es umgekehrt zur Positivkorrektur um die Frage, ob eine Substituierung der elterlichen Schulformwahlentscheidung durch die Empfehlung unter Rekurs auf die Vermeidung einer Überforderung der Schülerin bzw. des Schülers verfassungsrechtlich gerechtfertigt werden kann. Freilich gilt im Grundsatz insoweit Vergleichbares wie bei der Positivkorrektur und mithin ein Primat des Elternwillens, der von Verfassungs wegen im Interesse des Kindeswohls allenfalls in besonders gelagerten Ausnahmefällen staatlicherseits überwindbar ist.

\section{bb) Hemmung der Mitschüler ${ }^{*}$ innen}

Als grundsätzlich tauglicher Anknüpfungspunkt für die Rechtfertigung einer Negativkorrektur kommt aber der Rekurs auf die Grundrechtspositionen der Mitschüle$r^{*}$ innen in Betracht. Und in der Rechtswissenschaft wird dies in der Tat unter Hinweis darauf angenommen, dass (offensichtlich) ungeeignete/schwache Schüler*innen ihre Mitschülerinnen (empfindlich) in ihrem Lernerfolg behindern. Zur Vermeidung dessen sei die Negativkorrektur verfassungsrechtlich gerechtfertigt (vgl. etwa Avenarius \& Jeand'Heur, 1992, S. 45; Barczak, 2011, S. 177; Beaucamp, 2009, S. 281).

Indes finden sich in der Bildungsforschung keine Belege für diese Hemmungsthese; es fehlt - soweit ersichtlich - überhaupt an entsprechenden empirischen Untersuchungen (vgl. dazu auch Orth, 2011, S. 17). Diese dürften freilich auch nicht leicht durchzuführen sein. Erstens mag man davor zurückschrecken, die Schüler*innen zu „Versuchskaninchen“ zu machen, und zweitens wird es nicht leicht fallen, den Erfolg der Mitschüler*innen ohne die (mutmaßliche) Hemmung und Behinderung durch ungeeignete Schüler*innen zu simulieren. Letztlich wird man angesichts all dieser Ungewissheit - wie im abwehrrechtlichen Kontext auch sonst üblich - grundsätzlich eine Einschätzungsprärogative des Gesetzgebers akzeptieren müssen, welche vorliegend regelmäßig dazu führen wird, einen Verstoß gegen das Elterngrundrecht abzulehnen.

Je nach Ausgestaltung und Anwendung des jeweiligen Übergangsmodells kann es jedoch dazu kommen, dass auch unter Berücksichtigung der Einschätzungsprärogative des Gesetzgebers eine Geeignetheit zu verneinen und damit eine Unverhältnismäßigkeit anzunehmen ist. Dies ist der Fall, wenn die notwendige Prämisse, nämlich die Fähigkeit der Korrekturentscheidung, die Geeigneten von den Ungeeigneten $\mathrm{zu}$ unterscheiden und entsprechend zuzuordnen, nicht hinreichend garantiert wird. Ein solcher Fall liegt vor, wenn die nachfolgend näher zu untersuchenden sekundären Herkunftseffekte (siehe B. II. 3. b) oder andere leistungsverzerrende Faktoren die Grundschulempfehlung bestimmen. Ein leistungsverzerrender Effekt bei dem Erstellen der Übergangsempfehlung kann etwa dadurch eintreten, dass eine nur relative Einschätzung des Leistungsniveaus der Schülerin oder des Schülers im Verhältnis zu 
einer kleinen Vergleichsgruppe erfolgt, weil keine objektivierten, zentralen Maßstäbe angelegt werden. Insbesondere wenn die Grundschulempfehlung durch einen erfolgreich bestandenen Probeunterricht revidiert werden kann und sich herausstellt, dass der Probeunterricht eine auffällig hohe Erfolgsquote aufweist, stellt dies die Geeignetheit der Grundschulempfehlung nachhaltig in Frage.

\section{cc) Ergebnis}

Unter Ausblendung der Prognoseungenauigkeit der Übergangsempfehlungen und namentlich ihrer nachfolgend zu erörternden sozialen Selektivität lässt sich eine in Art. 6 Abs. 2 Satz 1 GG (Elternverantwortung) eingreifende Negativkorrektur unter Berufung auf die Interessen und Grundrechte der Mitschüler*innen rechtfertigen.

b) Verstoß gegen Art. 3 Abs. 3 Satz 1 6. Alt. GG wegen sekundärer Herkunftseffekte

\section{aa) Mittelbare Diskriminierung durch verbindliche Übergangsempfehlungen}

In der Bildungsforschung wird seit über 40 Jahren immer wieder gezeigt, dass Kinder aus bildungsfernen Elternhäusern häufig erst bei signifikant höheren Leistungswerten eine Gymnasialempfehlung erhalten als Kinder aus bildungsnahen Elternhäusern (näher dazu Barczak, 2011, S. 109 ff.; Bellenberg, 2012; Breidenstein, 2020).

Es finden sich verschiedene (kumulativ wirkende) Gründe zur Erklärung dieses Befundes. So gaben Lehrkräfte in einer Interview-Studie (Nölle, Höstermann, KrolakSchwerdt \& Gräsel, 2009) an, dass die von ihnen antizipierten elterlichen Unterstützungsmöglichkeiten die Vergabe der Übergangsempfehlung beeinflussen. In der Erziehungswissenschaft wird teils spekuliert, ob es gerechtfertigt oder gar rational sei, einem Kind aus einem bildungsnahen Elternhaus eher eine gymnasiale Empfehlung zu erteilen als einem im Hinblick auf die Leistungen vergleichbaren Kind aus einem bildungsfernen Elternhaus in der Jahrgangsstufe 4, da die Erfolgsaussichten aufgrund der zu erwartenden elterlichen Unterstützung regelmäßig höher seien (vgl. Autorengruppe Bildungsberichterstattung, 2020). Des Weiteren gaben Lehrkräfte in Interviews an, dass sie potentiellen Auseinandersetzungen mit Eltern über eine bevorstehende Übergangsempfehlung dadurch ausweichen, dass sie im Vorhinein eine entsprechende Anpassung der Noten vornehmen (Pohlmann-Rother, 2010). 
Übergänge in weiterführende Schulformen aus (verfassungs)rechtlicher Perspektive |

bb) Verfassungsrechtliche Würdigung

\section{(1) Verfassungsdogmatische Vorbemerkung}

Diese ,soziale Schieflage der Prognosen und Empfehlungen begründet eine mittelbare Diskriminierung, die, vorbehaltlich der sogleich zu behandelnden Zurechnungsfrage, verfassungsrechtlich von Art. 3 Abs. 3 Satz 1 6. Alt. GG als Diskriminierung nach der Herkunft eingefangen und vorbehaltlich einer Rechtfertigung zur Verfassungswidrigkeit des Übergangsmodells führt (zur Einbeziehung mittelbarer Diskriminierungen in Art. 3 Abs. 3 Satz 1 GG vgl. Heun, 2013, Rn. 117 m.w. N.; a. A. Rüfner, 1996, S. 333 ff.). Das Gruppenmerkmal der „Herkunft“ ist dabei als sozial-ökonomische, schichtenspezifische oder ständische Verwurzelung zu verstehen (vgl. Langenfeld, 2020, Rn. 60). Verstünde man den Herkunftsbegriff enger, läge eine ebenfalls rechtfertigungsbedürftige mittelbare Diskriminierung im Sinne von Art. 3 Abs. 1 GG vor. In diesem Falle würden sich die weitere Gedankenführung und das Ergebnis der Prüfung gleichwohl nicht unterscheiden.

\section{(2) (Keine) Zurechenbarkeit der Diskriminierung}

Zur Rechtfertigung wird zunächst vorgebracht, dass die durch Übergangsempfehlungen gegenüber Kindern aus bildungsfernen Elternhäusern regelmäßig bewirkten mittelbaren Diskriminierungen dem Gesetzgeber nicht zurechenbar seien. Zurechenbar sollen in Anlehnung an die Rechtsprechung des Bundesverfassungsgerichts (insb. BVerfGE 84, $239 \mathrm{ff}$.) sowie des Bundesverwaltungsgerichts zur Wehrpflicht (BVerwGE 122, 331) nur solche Benachteiligungen sein, die für den Gesetzgeber offensichtlich sind. Dies sei indes bei den schulgesetzlichen Regelungen der Bundesländer mit verbindlichen Übergangsempfehlungen nicht der Fall. Vielmehr trügen diese „die Gewähr ihrer gleichmäßigen Vollziehung in ausreichender Weise in sich“ und seien in Normenkomplexe eingebettet, die eine solche Vollziehung fördern (Barczak, 2011, S. $321 \mathrm{ff}$.). Wie sich diese „Behauptungen“ angesichts der geschilderten Ergebnisse der Bildungsforschung legitimieren können, ist nicht erkennbar. Vielmehr sind dem Normgeber durch eigene Normsetzung bewirkte und angesichts jahrzehntelanger Bildungsforschung seit Langem bekannte Vollzugsdefizite, welche im Sinne von Art. 3 Abs. 3 6. Alt. GG diskriminierend wirken, zurechenbar.

\section{(3) Kein Verstoß mangels Abhilfemöglichkeiten}

Des Weiteren hat Tristan Barczak gegen eine Verletzung von Art. 3 Abs. 3 Satz 1 6. Alt. GG eingewandt, dass keine gegenüber verbindlichen Übergangsempfehlungen überlegene Abhilfemöglichkeit im Hinblick auf die Ausräumung oder jedenfalls Abmilderung der Benachteiligung von Schüler*innen aus bildungsfernen Elternhäusern 
bestünde. Zum Beleg seiner These hat er eine Vielzahl von Abhilfemöglichkeiten erörtert und verworfen (vgl. Barczak, 2011, S. 325 ff.), ohne dass dem hier widersprochen werden soll. Anderes gilt allein für die von ihm verworfene Alternative einer unverbindlichen Empfehlung und mithin einem Wahlrecht der Eltern. Diesbezüglich wendet er ein, dass sich der Übertritt von der Primar- in die Sekundarstufe bei Freigabe des Elternwillens infolge der sozialschichtspezifischen Bildungserwartungen der Eltern ebenfalls sozial selektiv gestalte - mit entsprechend negativen Folgen für das Kindeswohl. Barczak nimmt an, dass bildungsferne Eltern bei freier (nur durch Empfehlungen angeleiteter) Übergangswahl mindestens genauso häufig wie verbindliche Übergangsempfehlungen eine Schulform wählen, die unterhalb des Begabungs- und Eignungsniveaus ihrer Kinder liegt. Aber selbst, wenn man einmal annimmt, dass bildungsferne Eltern nie nach oben abweichen, bleibt aus rechtlicher Perspektive die Frage, ob die geschilderte und dem Staat zurechenbare Diskriminierung unter Bezugnahme auf eine Ergebnisgleichheit im Falle diskriminierungsfreier privatautonomer Entscheidungsgewalt gerechtfertigt werden kann. Es bedarf wohl keiner näheren Begründung, dass die Antwort nur „Nein“ lauten kann. Eine staatlicherseits zu verantwortende, rechtsverbindliche Maßnahme kann nicht unter Hinweis darauf gerechtfertigt werden, dass grundrechtsfundierte privatautonome Entscheidungen (der Eltern) im statistischen Durchschnitt zu den gleichen Ergebnissen führen. Wegen der eng begrenzten Rechtfertigungsmöglichkeiten im Kontext der speziellen Diskriminierungsverbote nach Art. 3 Abs. 3 GG vermittelt auch die im Kontext von Art. 6 Abs. 2 Satz 1 GG bereits erörterte Hemmungshypothese keine ausreichende Rechtfertigung.

\section{cc) Ergebnis}

Verbindliche Übergangsempfehlungen im Sinne einer Negativkorrektur verletzen ob ihrer geschilderten sekundären Herkunftseffekte Art. 3 Abs. 3 Satz 1 6. Alt. GG (Diskriminierungsverbot aufgrund von Herkunft).

\section{Verfassungsmäßigkeit lediglich unverbindlicher Übergangsempfehlungen}

Wie eingangs angedeutet, wird in der Literatur auch die These vertreten, dass einem Kind der Zugang zu einer bestimmten Schulform der Sekundarstufe staatlicherseits von Verfassungs wegen versagt werden muss, wenn es die Mitschülerinnen andernfalls mit an Sicherheit grenzender Wahrscheinlichkeit empfindlich hemmen würde. Es wurde vorstehend nicht nur gezeigt, dass die Hemmungsthese empirisch nicht belastbar ist, sondern Übergangsempfehlungen zudem an strukturell bedingten Prognoseungenauigkeiten, insbesondere zu Lasten von Kindern aus bildungsfernen Elternhäusern, leiden. Soweit ein Landesgesetzgeber die Übergangsentscheidung also 
Übergänge in weiterführende Schulformen aus (verfassungs)rechtlicher Perspektive |

letztlich den Eltern überlässt und die Übergangsempfehlung lediglich unverbindlich ist, begegnet dieses keinen verfassungsrechtlichen Einwänden.

\section{Zusammenfassung}

Positiv korrigierende verbindliche Übergangsempfehlungen verletzen das Grundrecht der Eltern des unmittelbar betroffenen Kindes aus Art. 6 Abs. 2 Satz 1 GG. Das in Bremen vorfindliche Beratungsmodell mit Präklusionswirkung wirkt letztlich in diesem Sinne und ist daher insofern als verfassungswidrig einzustufen. Negativ korrigierende verbindliche Übergangsempfehlungen verletzen allein schon ob ihrer sekundären Herkunftseffekte Art. 3 Abs. 3 Satz 1 6. Alt. GG. Die Statuierung lediglich unverbindlicher Übergangsempfehlungen ist verfassungsrechtlich unbedenklich.

\section{Literatur und Internetquellen}

Hinweis der Redaktion: Als Hilfestellung sind die wesentlichen rechtlichen Regelungen, auf die in den Beiträgen des Heftes Bezug genommen wird, in einem das Heft begleitenden Online-Anhang abgedruckt. Er ist unter folgendem Link abrufbar: https://www.waxmann.com/ artikelART104608

Autorengruppe Bildungsberichterstattung. (2020). Bildung in Deutschland 2020. Ein indikatorengestützter Bericht mit einer Analyse zu Bildung in einer digitalisierten Welt. Bielefeld: wbv Media. Zugriff am 04.10.2021. Verfügbar unter: https://www.bildungsbericht. de/de/bildungsberichte-seit-2006/bildungsbericht-2020.

Avenarius, H., \& Jeand'Heur, B. (1992). Elternwille und staatliches Bestimmungsrecht bei der Wahl der Schullaufbahn. Berlin: Duncker \& Humblot. https://doi.org/10.3790/978-3428-47464-6

Barczak, T. (2011). Der Übergang von der Grundschule in die Sekundarstufe als Grundrechtsproblem. Baden-Baden: Nomos. https://doi.org/10.5771/9783845233291

Beaucamp, G. (2009). Neues zum Rechtsschutz gegen verbindliche Schulwahlempfehlungen. Neue Zeitschrift für Verwaltungsrecht, 28 (5), 280-283.

Bellenberg, G. (2012) Bildungsgerechtigkeit beim Übergang von der Grundschule in die Sekundarstufe I. Empirische Befunde zum Prozess der Übergangsentscheidung. In Institut für Bildungsforschung und Bildungsrecht e.V. (Hrsg.), Selektion und Gerechtigkeit in der Schule (S. 59-78). Baden-Baden: Nomos.

Bellenberg, G., \& Tillmann, K.-J. (2011). Schulnoten - Elternrecht - Probeunterricht. Friedrich Jahresheft, 29, 61-65.

Breidenstein, G. (2020). Ungleiche Grundschulen und die meritokratische Fiktion im deutschen Schulsystem. Zeitschrift für Grundschulforschung, 13, 295-307. https://doi. org/10.1007/s42278-020-00078-4

Frotz, J. (2006). Die Reichweite des grundrechtlichen Abwehranspruchs. Berlin: Logos.

Heun, W. (2013). Art. 3 GG. In: H. Dreier (Hrsg.), Grundgesetz-Kommentar (Bd. 1). Tübingen: Mohr Siebeck.

Hölbling, P. (2010). Wieviel Staat vertragen Eltern? Systematische Entfaltung eines gestuften Maßnahmenkonzepts vor dem Hintergrund des Elterngrundrechts. Berlin: Duncker \& Humblot. https://doi.org/10.3790/978-3-428-53347-3 
Huster, S., \& Rux, J. (2020). Art. 20 GG. In V. Epping \& C. Hillgruber (Hrsg.), Beck'scher Online-Kommentar Grundgesetz. München: C. H. Beck.

Institut für Bildungsforschung und Bildungsrecht e. V. (Hrsg.). (2012). Selektion und Gerechtigkeit in der Schule. Baden-Baden: Nomos.

Jeand'Heur, B. (1993). Verfassungsrechtliche Schutzgebote zum Wohl des Kindes und staatliche Interventionspflichten aus der Garantienorm des Art. 6 Abs. 2 GG. Berlin: Duncker \& Humblot. https://doi.org/10.3790/978-3-428-47857-6

Langenfeld, C. (2020). Art. 3 Abs. 3 GG. In T. Maunz \& G. Dürig (Begr.), Grundgesetz-Kommentar. München: C.H. Beck.

Maaz, K., Hasselhorn, M., Idel, T.-S., Kieme, E., Lütje-Klose, B., Stanat, P., et al. (Hrsg.). (2019). Zweigliedrigkeit und Inklusion im empirischen Fokus. Ergebnisse der Evaluation der Bremer Schulreform. Münster: Waxmann.

Meinel, F. (2007). Lebensentscheidungen auf ungewisser Grundlage. Die Öffentliche Verwaltung, 60 (2), 66-71.

Nölle, I., Höstermann, T., Krolak-Schwerdt, S., \& Gräsel, C. (2009). Relevante diagnostische Informationen bei der Übergangsempfehlung. Die Perspektive der Lehrkräfte. Unterrichtswissenschaft, 37, 294-319.

Orth, J. (2011). Verfassungsrechtliche Anforderungen an die Schulstruktur. Neue Zeitschrift für Verwaltungsrecht, 30 (1), 14-19.

Pohlmann-Rother, S. (2010). Die Herausbildung der Übergangsempfehlung am Ende der Grundschulzeit. Zeitschrift für Grundschulforschung, 3 (2) 136-148.

Roth, W. (2003). Die Grundrechte Minderjähriger im Spannungsfeld selbständiger Grundrechtsausübung, elterlichen Erziehungsrechts und staatlicher Grundrechtsbindung. Berlin: Duncker \& Humblot. https://doi.org/10.3790/978-3-428-51000-9

Rüfner, W. (1996). Die mittelbare Diskriminierung und die speziellen Gleichheitssätze in Art. 3 Abs. 2 und 3 GG. In R. Wendt (Hrsg.), Staat, Wirtschaft, Steuern. Festschrift für Karl Heinrich Friauf zum 65. Geburtstag (S. 331-341). Heidelberg: Müller.

Scholz, R. (2020). Art. 12 GG. In T. Maunz \& G. Dürig (Begr.), Grundgesetz-Kommentar. München: C.H. Beck.

Tettinger, P. (2005). Zukunftssicherung durch Bildung der Kinder. Nordrhein-Westfälische Verwaltungsblätter, 19, 332-337.

Wolfram Cremer, Prof. Dr., geb. 1963, Lehrstuhl für Öffentliches Recht und Europarecht, Ruhr-Universität Bochum

E.Mail: wolfram.cremer@ruhr-uni-bochum.de

Antonia Tobisch, Dr., geb. 1990, Lehrstuhl für Öffentliches Recht und Europarecht, Ruhr-Universität Bochum

E-Mail: antonia.tobisch@ruhr-uni-bochum.de

Korrespondenzadresse: Ruhr-Universität Bochum, Lehrstuhl für Öffentliches Recht und Europarecht, Universitätsstraße 150, 44801 Bochum 